\title{
Perfil dos pacientes cadastrados com hanseníase no centro clínico universitário em Patos de Minas
}

\section{Leprosy patients profile registered in the university clinical center in Patos de Minas}

\author{
Monique Martins' ${ }^{1}$ Lyza Alencar Siqueira' ${ }^{1}$, Cicero Augusto Zolli², \\ Natália de Fátima Gonçalves Amâncio ${ }^{3}$
}

Martins M, Siqueira LA, Zolli CA, Amâncio NFG. Perfil dos pacientes cadastrados com hanseníase no centro clínico universitário em Patos de Minas / Leprosy patients profile registered in the university clinical center in Patos de Minas. Rev Med (São Paulo). 2019 set.-out.;98(5):304-8.

RESUMO: a hanseníase é uma doença infectocontagiosa causada pelo mycobacterium leprae e se manifesta principalmente através de sinais e sintomas dermatoneurológicos. O objetivo do estudo foi descrever o perfil clínico e epidemiológico dos portadores de hanseníase cadastrados no centro clínico universitário. Foi elaborado um roteiro de entrevista para 33 pacientes referentes às condições socioeconômicas, demográficas, ambientais e clínicas. A maioria era adultos, (média de 61,7 anos), pardos, sexo masculino, baixa escolaridade, economicamente ativos, todos classificados como baixa renda. Entre as formas da doença, a mais prevalente foi a dimorfa com a classificação operacional multibacilar. A incapacidade física de grau 0 predominou com $66,7 \%$. Dentre os fatores que proporcionam maior risco à saúde, inclui-se a idade avançada e a baixa condição socioeconômica. A predominância multibacilar demonstra a importância do diagnóstico precoce e tratamento adequado por se tratar de caráter mais agressivos, que juntamente com as reações são importantes causas de incapacidade física, acarretando absenteísmo do trabalho.

Descritores: Hanseníase; Hanseníase dimorfa; Hanseníase multibacilar/diagnóstico; Planejamento socioeconômico; Brasil/ epidemiologia.

\begin{abstract}
: leprosy is an infectious disease caused by mycobacterium leprae and manifests mainly through dermatoneurological signs and symptoms. The objective of this study was to describe the clinical and epidemiological profile of leprosy patients enrolled at the university clinical center. An interview script was prepared for 33 patients regarding socioeconomic, demographic, environmental and clinical conditions. The majority were adults, (average 61.7 years), brown, male, low educational level, economically active, all classified as low income. Among the forms of the disease, the most prevalent was boderline with multibacillary operational classification. The grade 0 disabilities predominated with $66.7 \%$. The advanced age and the socioeconomic profile of the majority of the patients, constitute in greater risks to the health. Multibacillary predominance demonstrates the importance of early diagnosis and appropriate treatment for being more aggressive, which together with the reactions are important causes of physical incapacity, leading to absenteeism of work.
\end{abstract}

Keywords: Leprosy; Leprosy, borderline; Leprosy, multibacillary/ diagnosis; Socioeconomic planning; Brazil/epidemiology.

Trabalho realizado no Centro Clínico Universitário de Patos de Minas do Centro Universitário de Patos de Minas (UNIPAM), Patos de Minas, MG.

1. Acadêmico de medicina do Centro Universitário de Patos de Minas (UNIPAM), Patos de Minas, MG. ORCID: Martins M - https://orcid.org/00000001-6798-9755; Siqueira LA - https://orcid.org/0000-0001-9724-1289. Email: moniquemartins7@outlook.com, lyzaalencar@gmail.com.

2. Médico Dermatologista pelo Hospital das Clínicas de São Paulo (USP); Título de especialista em dermatologia pela Sociedade Brasileira de Dermatologia, São Paulo, SP. ORCID: https://orcid.org/0000-0002-6815-6111. Email: cicerozollidermato@gmail.com.

3. Docente no Centro Universitário de Patos de Minas (UNIPAM); Doutora em Promoção de Saúde, Patos de Minas, MG. ORCID: https://orcid. org/0000-0002-7716-6602. Email:nfga@unipam.edu.br.

Endereço para correspondência: Monique Martins. Rua Major Gote, 944 apto 504 - Alto dos Caiçaras. Patos de Minas, MG, Brasil. CEP: $38702-054$.

Email: moniquemartins7@outlook.com. 


\section{INTRODUÇÃO}

A hanseníase é uma doença infectocontagiosa causada pelo Mycobacterium leprae, de evolução lenta, que se manifesta principalmente através de sinais e sintomas dermatoneurológicos nos olhos, mãos e pés $^{1,2}$. O Ministério da Saúde classifica a hanseníase como indeterminada, tuberculóide, dimorfa e virchoviana, sendo respectivamente as duas primeiras formas paucibacilares (poucos bacilos estão presentes) e as duas últimas multibacilares ${ }^{3}$.

O Mycobacterium leprae não acomete somente a pele, mas também o sistema nervoso periférico, causando a perda de sensibilidade, atrofias, paresias e paralisias musculares que, se não diagnosticadas e tratadas precocemente, podem evoluir para incapacidades físicas permanentes, tornando assim a hanseníase uma doença de grande importância na saúde pública ${ }^{4}$.

Segundo a Organização Mundial da Saúde (OMS), 94\% dos casos de hanseníase notificados em 2014 pertenciam a 13 países, grupo em que o Brasil está incluso. Junto a isso, essa organização ainda informa que o Brasil em conjunto com Índia e Indonésia notificam mais de 10.000 novos pacientes/ano, representando $81 \%$ dos pacientes recém diagnosticados e notificados no mundo ${ }^{5}$.

O Brasil ocupa o segundo lugar na prevalência mundial de hanseníase em relação a casos novos, e no âmbito das américas é responsável por mais de $92 \%$ do número de casos registrados 6 . A OMS preconiza como meta de eliminação da hanseníase como menos de um caso para cada dez mil habitantes. A população brasileira apresentou coeficiente de prevalência de 1,56 casos por 10.000 habitantes em 20107. Já em 2016, o coeficiente geral de detecção de 12,2 casos por 100.000 habitantes, o qual sofreu uma redução em relação a $2010(18,2)$, porém continua sendo classificado como país de alta carga para a doença. As regiões Norte e Centro-Oeste persistem como áreas endêmicas ${ }^{7,8}$.

De acordo com o Ministério da Saúde, os valores médios dos coeficientes no período de 2012 a 2016 apresentaram predominância do sexo masculino em relação ao feminino. Em relação ao nível de escolaridade analisado nesse período, houve prevalência no segmento analfabeto + ensino fundamental incompleto (55\%). Quando se observa o fator raça/cor de 2012-2016, a parda representa $58,9 \%$, a branca $26,8 \%$, a preta $12,8 \%$, a amarela $0,9 \%$ e a indígena $0,4 \%$. Nessa mesma faixa de tempo, observou-se que a proporção de casos novos multibacilar foi prevalente na população masculina $(62,7 \%)$ e de paucibacilar na população feminina $(58,6 \%)^{8}$.

A hanseníase, quando não diagnosticada e tratada de forma precoce e adequada, evolui para incapacidades e deformidades físicas, as quais levam à diminuição da capacidade de trabalho, limitação da vida social e problemas psicológicos 9 . O grau de incapacidade é determinado a partir da avaliação neurológica dos olhos, mãos/pés e tem seu resultado expresso em valores que variam de 0 (zero) a II (dois) ${ }^{1}$. Assim, grau 0 é determinado quando não há incapacidade nos olhos, nas mãos e nos pés; grau 1 quando há incapacidade (diminuição ou perda de sensibilidade nos olhos, nas mãos ou nos pés) e grau 2 quando há incapacidade e deformidade nos olhos, nas mãos e nos pés ${ }^{2}$. O acometimento neural pode ocorrer em todas as formas da hanseníase, mostrando que a avaliação e registro das incapacidades são importantes para evitar a instalação de incapacidades durante o tratamento ${ }^{2}$.

Em 2016 o Brasil ainda é classificado como alta carga para a hanseníase, sendo o segundo com a maior incidência no mundo ${ }^{2}$. Em Minas Gerais, Patos de Minas ocupa a $19^{\circ}$ posição, acima das metas estabelecidas pela $\mathrm{OMS}^{10}$. Dessa forma, o objetivo foi identificar o perfil e as formas clínicas desses pacientes, para melhor prevenção da comunidade e diminuição da incidência da doença.

\section{MÉTODOS}

Trata-se de um estudo transversal, descritivo, com abordagem quantitativa. Formaram a amostra deste estudo os pacientes portadores de hanseníase, atendidos pelo Centro Clínico Universitário, no Município de Patos de Minas - Minas Gerais, no período de março a dezembro de 2018. Para a análise das formas clínicas de hanseníase foi adotada a classificação de Madri (1953) definida no VI Congresso Internacional de Leprologia que orienta como formas clínicas: indeterminada (I), tuberculóide (T), dimorfa (D) e virchowiana (V) ${ }^{1}$. Para a coleta dos dados referentes às condições socioeconômicas, demográficas, ambientais e clínicas, foi utilizado um roteiro de entrevista contendo questões fechadas. A identificação das incapacidades físicas e a determinação do grau das mesmas foram realizadas a partir do exame físico dos olhos, mãos e pés. Tais exames seguiram os parâmetros adotados pelo Ministério da Saúde do Brasil.

O estudo foi submetido à aprovação pelo Comitê de Ética e Pesquisa (CEP) do UNIPAM, por meio do parecer consubstanciado de número 2.512.667. Os critérios de inclusão foram todos os pacientes com hanseníase que buscaram atendimento dermatológico no centro clínico universitário no período estudado. Todos aceitaram participar da pesquisa. Os critérios de exclusão foram os pacientes encaminhados com outras doenças. A amostragem foi por conveniência. Todo paciente que chegava ao centro clínico no período de março a dezembro de 2018 pôde, teoricamente, participar da amostra, desde que o mesmo preenchia os critérios de inclusão da pesquisa. Após busca ativa dos pacientes e análise dos critérios de inclusão 33 pacientes diagnosticados com hanseníase formaram a amostra deste estudo. 


\section{RESULTADOS}

A prevalência da doença foi maior na faixa etária entre 50 a 98 anos, com idade média de 61,7 anos, representando 73. Em relação ao sexo, $76 \%$ dos pacientes portadores de hanseníase eram do sexo masculino e $24 \%$ do sexo feminino.

Houve predominância da cor parda com 57,6\%, seguida pelos pacientes da cor branca com $39,4 \%$ e com menor percentual, $3 \%$ da cor negra.

Dentre as ocupações identificadas, as mais frequentes foram aposentados, 27,3\%, motoristas e lavradores com $12 \%$, pedreiros $4 \%$ e outras profissões somaram $39,7 \%$. A renda familiar no geral foi baixa, com $63,7 \%$ na família com renda de 1 a 3 salários mínimos, seguida de $23,7 \%$ com menos de um salário, nenhum paciente com renda maior que 4 salários e $9 \%$ não souberam informar (Figura 1).

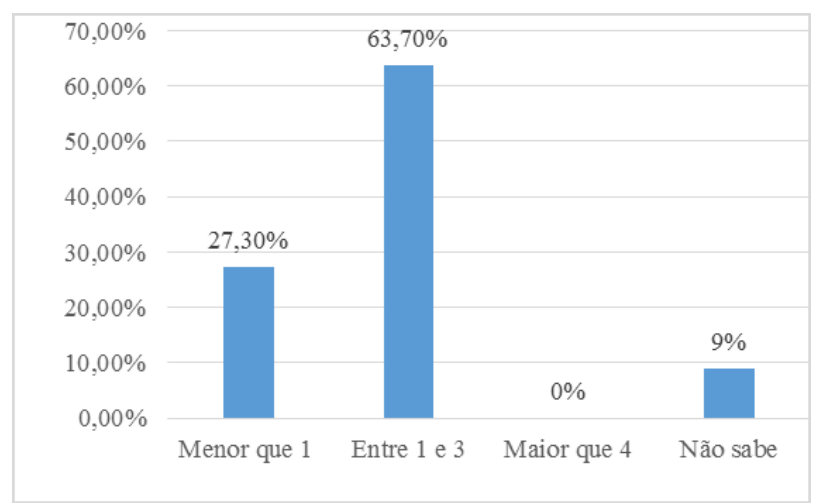

Figura 1: Distribuiçao de renda dos participantes por salário mínimo

De acordo com o grau de escolaridade, em sua maioria, 72,7\% dos pacientes possuíam até o $1^{\circ}$ grau do ensino fundamental, seguido de $18 \%$ de segundo grau completo e $9 \%$ eram analfabetos. O local de moradia da maioria dos pacientes foi na zona urbana (88\%), restando apenas $12 \%$ para os que moram na zona rural. Apenas 18,2\% dos pacientes relataram casos na família de hanseníase e $81,8 \%$ não confirmaram.

Dos 33 pacientes, 9 estavam realizando a poliquimioterapia (PQT) e 24 tinham reação após o tratamento e por isso estavam realizando acompanhamento na clínica de especialidades. Com relação às formas clínicas da hanseníase, a maioria dos pacientes apresentou a classificação multibacilar, sendo a forma dimorfa a mais prevalente, com $66,7 \%$ dos casos, seguido da virchowina com $11,1 \%$, já a classificação paubacilar apresentou apenas a forma tuberculóide com 22,2\% dos casos (Figura 2).

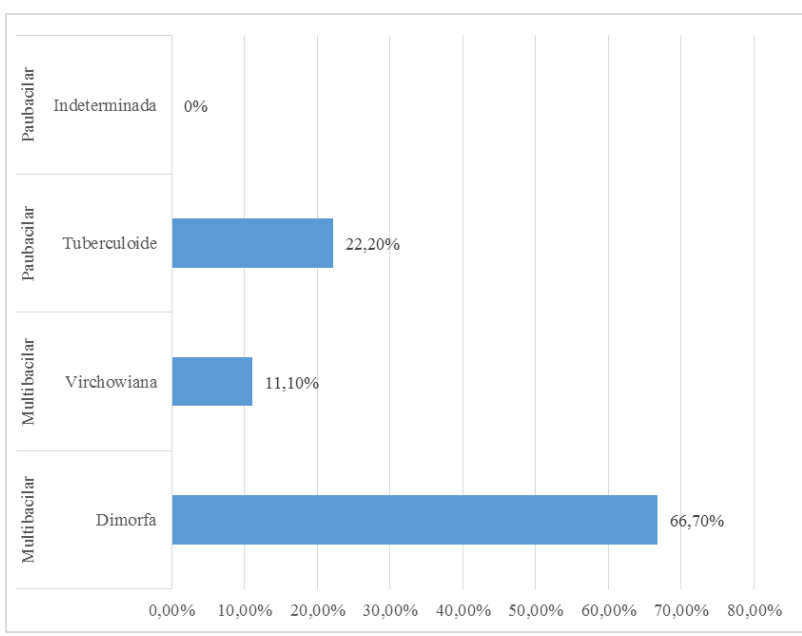

Figura 2: Distribuição da forma clínica de Hanseníase nos participantes da pesquisa

Os pacientes foram avaliados quanto ao grau de incapacidade de acordo com o Ministério da Saúde em três graus $(0,1,2)$. Os graus são atribuídos para cada mão, olho e pé, o maior valor atribuído, representa o maior grau de incapacidade do indivíduo. $\mathrm{Na}$ análise do grau de incapacidade durante o período do estudo, verificou-se que $66,7 \%$ dos pacientes não apresentaram incapacidade. Em contrapartida $22,2 \%$ dos pacientes apresentaram grau 1 de incapacidade, e $11,1 \%$ grau 2 . Os doentes que tinham algum grau de incapacidade apresentavam a forma multibacilar, visto que a forma dimorfa prevaleceu na incapacidade grau 1 e a forma virchowiana no grau 2 (Figura 3). Durante o tratamento da PQT 55,6\% dos pacientes tiveram reação do tipo $1 ; 33,3 \%$ sem nenhuma reação e $11,1 \%$ com reação tipo 2 .

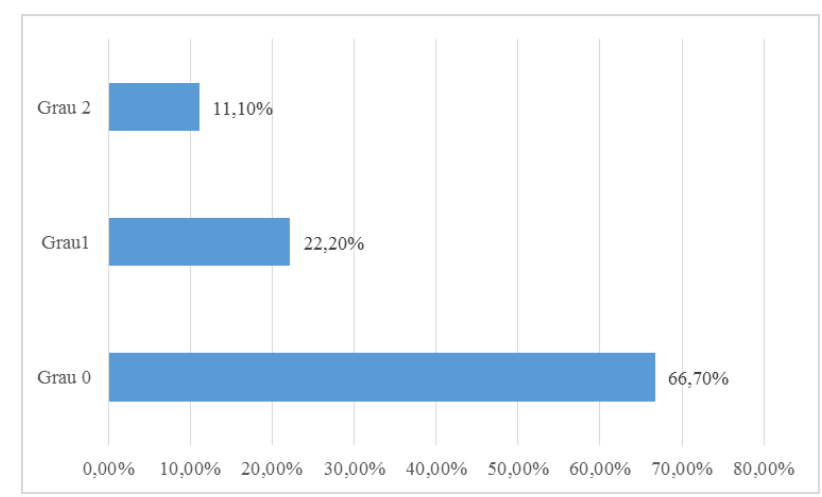

Figura 3: Avaliação do grau de incapacidade dos participantes da pesquisa

\section{DISCUSSÃO}

Com o intuito de descrever o perfil clínico e 
epidemiológico dos portadores de hanseníase cadastrados no Centro Clínico Universitário de Patos de Minas, o presente estudo observou que a faixa etária média dos pacientes com hanseníase foi de 61,7 anos, fato que contrasta com outros estudos, em que a prevalência da doença é maior na população ativa, na faixa dos 13 a 49 anos $^{11,12,13}$. Junto a isso, quando se analisou a prevalência da doença entre os sexos, encontrou uma maior preponderância no sexo masculino (76\%). Já no estudo de Batista et al. ${ }^{14}$ não houve diferença significativa entre os sexos, diferentemente da OMS que relata ter uma preponderância entre o gênero masculino na proporção de 2:1. Alguns estudos podem ter predominância do sexo feminino, devido a menor preocupação com o corpo e estética do sexo masculino.

Em relação a cor da pele, encontrou-se predomínio da cor parda $(57,6 \%)$, seguida da branca $(39,4 \%)$ e negra $(3 \%)$. Essa prevalência também foi encontrada em outro estudo $^{15}$. Contudo, em outros a cor branca prevaleceu ${ }^{12,13}$. Dessa forma, deve-se levar em consideração o local e a etnia onde foi realizado o estudo. Além disso, pela história de miscigenação do brasileiro é provável que a cor parda predomine.

Ao observar as ocupações, notou-se maior presença de aposentados (27,3\%), seguida de motoristas (12\%), lavradores $(12 \%)$ e pedreiros $(4 \%)$. Estes resultados contrastam com outros autores que encontraram lavrador e autônomo em sua maioria ${ }^{13,16}$. Porém há a semelhança de serem trabalhos pouco remunerados, o que justifica a baixa renda da maioria dos pacientes em todos estudos pesquisados e também no presente estudo. Além disso, a maioria dos entrevistados possuía grau de escolaridade até o $1^{\circ}$ grau do ensino fundamental $(72,7 \%)$. Da mesma forma, estudos detectaram grau de escolaridade baixo dos pacientes com hanseníase, fato que possui grande relevância para o planejamento das ações de educação em saúde e sensibilização da comunidade ${ }^{16,17}$.

Quando contestados a respeito de casos na família, apenas $18,2 \%$ responderam que sim. Contudo, esses casos estavam relacionados com a faixa etária abaixo de 15 anos, o que demonstra a alta capacidade de transmissão da doença, ocasionada muitas vezes, entre os familiares ${ }^{13}$.

A classificação mais encontrada foi a multibacilar com prevalência da forma clínica dimorfa $(66,7 \%)$, seguida da virchowiana $(11,1 \%)$, e em relação a classificação

\section{REFERÊNCIAS}

1. Brasil. Ministério da Saúde. Secretaria de Políticas de Saúde. Departamento de Atenção Básica. Guia para o controle da hanseníase. Brasília; 2002. (Série A. Normas e Manuais Técnicos; n. 111). Disponível em: http://bvsms.saude.gov. br/bvs/publicacoes/guia_de_hanseniase.pdf.

2. Brasil. Ministério da Saúde. Guia prático de hanseníase. Brasília; 2017. Disponível em: http://portalarquivos2.saude. gov.br/images/pdf/2017/novembro/22/Guia-Pratico-deHanseniase-WEB.pdf. paucibacilar encontrou-se apenas a forma tuberculóide (22,2\%). Do contrário, em uma revisão de literatura a forma mais prevalente foi a tuberculóide com 50,4\% e a classificação operacional foi a paubacilar com $54,3 \%{ }^{12}$. Essa classificação é muito importante, pois ela analisa o número das lesões cutâneas, permitindo o tratamento em locais que não tem estrutura para realizar a baciloscopia.

Nesse estudo, percebeu-se que mais da metade dos pacientes não apresentaram incapacidade $(66,7 \%)$, $22,2 \%$ apresentaram grau 1 e $11,1 \%$ grau 2 . Os principais problemas da hanseníase ocorrem devido ao grau de incapacidade causado principalmente pela lesão neural, o que limita as atividades diárias do paciente, por isso é importante analisar a incapacidade no período de tratamento da doença.

O estado reacional da hanseníase é caracterizado pela resposta imunológica ao bacilo, podendo ser classificado como tipo 1, na qual ocorre exacerbação das lesões prévias, e tipo 2, em que há desenvolvimento de nódulos eritematosos e febre. No presente estudo, grande parte dos pacientes tiveram reação tipo $1(55,6 \%)$ e $11,1 \%$ tiveram reação tipo 2 . No estudo de Souza ${ }^{18}$, o autor mostra que a maioria das reações dos seus participantes foram do tipo 2 e de 118 pacientes em estado reacional, 66 apresentavam a forma virchowiana.

\section{CONCLUSÕES}

A hanseníase é uma doença que existe há séculos, porém ainda é muito frequente e tem alto poder incapacitante, se tornando um problema de saúde pública. O Brasil ainda é um dos principais países com maior frequência desta doença. Dessa forma, o Ministério da saúde preconiza uma prevalência menor que 1 caso por 10.000 habitantes e uma incidência menor que 2 casos por 100.000 habitantes. Patos de Minas ainda não se enquadra em ambos os parâmetros, mesmo com o tratamento da poliquimioterapia vigente. Além disso, a maioria dos pacientes estudados apresentavam reações, o que causa maior período de abstinência ao trabalho e prejuízo nas atividades do portador da doença. Portanto, é importante ressaltar a importância da educação em saúde para informar e esclarecer a população para assim auxiliar na detecção precoce e tratamento adequado da doença.

3. Lima HMN. Perfil epidemiológico dos pacientes com hanseníase atendidos em Centro de Saúde em São Luís, MA. Rev Bras Clin Med. 2010;8(4):323-7. Disponível em: http://files.bvs.br/ upload/S/1679-1010/2010/v8n4/a007.pdf.

4. Brasil. Ministério da Saúde. Manual de prevenção de incapacidades/ elaboração: área técnica de dermatologia sanitária. Brasília; 2001. Disponível em: http://bvsms.saude.gov.br/bvs/publicacoes/ MANPREV2000.pdf.

5. World Health Organization. Global leprosy strategy: accelerating 
towards a leprosy-free world. Geneva; 2016 [cited 2019 June 20]. Available from: https://apps.who.int/iris/bitstream/hand le/10665/208824/9789290225201-pt.pdf;jsessionid=8a298c 92164 fecaa 7 cb5159feef54679? sequence $=17$.

6. Brasil. Ministério da Saúde. Estratégia nacional para o enfrentamento da hanseníase - 2019-2022. Brasília; 2019 [citado 20 jun. 2019]. Disponível em: http://portalarquivos2. saude.gov.br/images/pdf $/ 2019 / \mathrm{marco} / 27 /$ estrategia-nacionalcghde-consulta-publica-27mar.pdf.

7. Alves ED, Ferreira TL, Nery I; NESPROM. Hanseníase: avanços e desafios. Brasília; 2014. Disponível em: http:// www.morhan.org.br/views/upload/hanseniaseavancoes.pdf.

8. Brasil. Ministério da Saúde. Secretaria de Vigilância em Saúde. Caracterização da situação epidemiológica da hanseníase e diferenças por sexo, Brasil, 2012-2016. Bol Epidemiol. 2018;49(4):1-10. Disponível em: http://portalarquivos2.saude. gov.br/images/pdf/2018/janeiro/31/2018-004-hanseniasepublicacao.pdf.

9. Lana FCF, Amaral EP, Franco MS, Lanza FM. Estimativa da prevalência oculta da hanseníase no Vale do Jequitinhonha MG. Rev Min Enferm. 2004;8(2):295-300. Disponível em: http://www.reme.org.br/artigo/detalhes/740.

10. Brasil. Ministério da Saúde. DATASUS Tecnologia da Informação a Serviço do SUS. Acompanhamento dos dados de hanseníase em Minas Gerais: Brasil 2000-2016/2017: banco de dados do Sinan/SVS-MS [citado 11 jul. 2018]. Disponível em: http://tabnet.datasus.gov.br/cgi/webtabx. exe?Hanseniase/hantfbr17.def.

11. Melão S, Blanco FLO, Mounzer N, Veroneze CCD, Simões PWTA. Perfil epidemiológico dos pacientes com hanseníase no extremo sul de Santa Catarina, no período de 2001 a 2007. Rev Soc Bras Med Trop. 2011;44(1):79-84. http://dx.doi. org/10.1590/S0037-86822011000100018.
12. Veloso DS, Melo CB, Sá TLB, Santos JP, Nascimento EF, Costa FAC. Perfil clínico epidemiológico da hanseníase: uma revisão integrativa. Rev Eletr Acervo Saúde. 2018;10:142937. doi: 10.25248/REAS146_2018.

13. Batista ES, Campos RX, Queiroz RCG, et al. Perfil sóciodemográfico e clínico-epidemiológico dos pacientes diagnosticados com hanseníase em Campos dos Goytacazes, RJ. Rev Bras Clin Med. 2011;9(2):101-6. Disponível em: http://files.bvs.br/upload/S/1679-1010/2011/v9n2/a1833.pdf.

14. World Health Organization. Global leprosy situation. Weekly Epidemiol Record. 2010;85(35):337-48. Available from: http:/www.who.int/wer/2010/wer8535.pdf.

15. Sarmento APA, Pereirão AM, Ribeiro F, Castro JL, Almeida MB, Ramos NM. Perfil epidemiológico da hanseníase no período de 2009 a 2013 no município de Montes Claros (MG). Rev Soc Bras Clin Med. 2015;13(3):180-4. Disponível em: http://files.bvs.br/upload/S/1679-1010/2015/v13n3/a5389. pdf.

16. Aquino DMC, Caldas AJM, Silva AAM, Costa JML, et al. Perfil dos pacientes com hanseníase em área hiperendêmica da Amazônia do Maranhão, Brasil. Rev Soc Bras Med Trop. 2003;36(1):57-64. http://dx.doi.org/10.1590/S003786822003000100009 .

17. Miranzi SSC, Pereira LHM, Nunes AA. Perfil epidemiológico da hanseníase em um município brasileiro, no período de 2000 a 2006. Rev Soc Bras Med Trop. 2010;43(1):62-67. http:// dx.doi.org/10.1590/S0037-86822010000100014.

18. Souza LWF. Reações hansênicas em pacientes em alta por cura pela poliquimioterapia. Rev Soc Bras Med Trop. 2010;43(6):737-9. http://dx.doi.org/10.1590/S003786822010000600029 .

Recebido: 02.04.19

Aceito: 30.07 .19 